\title{
Statefinder diagnostic in a torsion cosmology
}

\author{
Xin-zhou Li, Chang-bo Sun and Ping Xi \\ Shanghai United Center for Astrophysics(SUCA), Shanghai Normal University, 100 \\ Guilin Road, Shanghai 200234,China \\ E-mail: kychz@shnu.edu.cn
}

\begin{abstract}
We apply the statefinder diagnostic to the torsion cosmology, in which an accounting for the accelerated universe is considered in term of a Riemann-Cartan geometry: dynamic scalar torsion. We find that there are some typical characteristic of the evolution of statefinder parameters for the torsion cosmology that can be distinguished from the other cosmological models. Furthermore, we also show that statefinder diagnostic has a direct bearing on the critical points. The statefinder diagnostic divides the torsion parameter $a_{1}$ into differential ranges, which is in keeping with the requirement of dynamical analysis. In addition, we fit the scalar torsion model to ESSENCE supernovae data and give the best fit values of the model parameters.
\end{abstract}

PACS numbers: 98.80.-k, 98.80.Es 


\section{Introduction}

The current observations, such as SNeIa (Supernovae type Ia), CMB (Cosmic Microwave Background) and large scale structure, converge on the fact that a spatially homogeneous and gravitationally repulsive energy component, referred as dark energy, accounts for about $70 \%$ of the energy density of universe. Some heuristic models that roughly describe the observable consequences of dark energy were proposed in recent years, a number of them stemming from a certain physics [1] and the others being purely phenomenological [2]. Dark energy can even behave as a phantom and effectively violate the weak energy condition[3].

In various cosmological models, fundamental quantities are either geometrical (if they are constructed from a spacetime geometry directly) or physical (if they depend upon physical fields). Physical quantities are certainly model-dependent, while geometrical quantites are more universal. About thirty years ago, the bouncing cosmological model with torsion was suggested in Ref. [4], but the torsion was imagined as playing role only at high densities in the early universe. Goenner et al. made a general survey of the torsion cosmology [5], in which the equations for all the PGT (Poincaré Gauge Theory of gravity) cases were discussed although they only solved in detail a few particular cases. Recently some authors have begun to study torsion as a possible reason of the accelerating universe [6]. Nester and collaborators [7] consider an accounting for the accelerated universe in term of a Riemann-Cartan geometry: dynamic scalar torsion. They explore the possibility that the dynamic PGT connection, reflecting the nature of dynamic PGT torsion, provides the accelerating force. With the usual assumptions of homogeneity and isotropy in cosmology and specific cases of the suitable parameters and initial conditions, they find that torsion field could play a role of dark energy.

One of the motivation was to avoid singularities in the initial investigations of torsion cosmology [8]. However, it soon was found that non-linear torsion effects were more likely to produce stronger singularities [9]. The non-linear effects turn out to play a key role for the outstanding present day mystery: the accelerated universe. In the various PGT, the connection dynamics decomposed into six modes with certain spin and parity: $2^{ \pm}, 1^{ \pm}, 0^{ \pm}$. Some investigations showed that $0^{ \pm}$may well be the only acceptable dynamic PGT torsion modes [10]. The pseudoscalar mode $0^{-}$is naturally driven by the intrinsic spin of elementary fermions, therefore it naturally interacts with such sources. Consequently, it is generally thought that axial torsion must be small and have small effects at the late time of cosmological evolution. This is a major reason why one does not focus on this mode at the late time. On the other hand, the scalar mode $0^{+}$does not interact in any direct obvious fashion with any known type of matter [11], therefore one can imagine it as having significant magnitude and yet not being

conspicuously noticed. Furthermore, there is a critical non-zero value for the affine scalar curvature since $0^{+}$mode can interact indirectly through the non-linear equations. The homogeneity and isotropy of cosmology have received strong confirmation from modern observations, which greatly restrict the possible types of non-vanishing fields. 
Under the assumption of homogeneity and isotropy, $0^{+}$mode has only a time component and it can be specified as the gradient of a time-dependent function. Therefore, the cosmological models with the scalar mode offer a situation where dynamic torsion may lead to observable effect at late time. We emphasize again that one does not focus on the early universe, where one could indeed expect large effects (though their signature would have to be separated from other large effects), and substitutionally asks about traces of torsion effects at the late time of cosmological evolution [7].

Obviously, the fine-tuning problem is one of the most important issues for the torsion cosmology [7]. And a good model should limit the fine-tuning as much as possible. The dynamical attractor of the cosmological system has been employed to make the later time behaviors of the model insensitive to the initial condition of the field and thus alleviates the fine-tuning problem [12]. Furthermore, Nester et al [7] have shown that the Hubble parameter and $\ddot{a}$ have an oscillatory form for the scalar torsion cosmology.

The traditional geometrical parameters, i.e., the Hubble parameter $H \equiv \dot{a} / a$ and the deceleration parameter $q \equiv-\ddot{a} a / \dot{a}^{2}$, are two elegant choices to describe the expansion state of universe but they can not distinguish various accelerating mechanism uniquely, because a quite number of models may just correspond to the same current values of $H$ and $q$. However, Sahni, Saini, Starobinsky and Alam [13] have introduced the statefinder pair $\{r, s\}: r \equiv \dddot{a} / a H^{3}, s \equiv(r-1) / 3(q-1 / 2)$. It is obviously a natural next step beyond $H$ and $q$. Fortunately, as is shown in the literatures [14, 15], the statefinder parameters which are also geometrical diagnostics, are able to differentiate a series of cosmological models successfully. Using the discussion of statefinder parameters in the scalar torsion cosmology, we explain easily why the present field equations modify the expansion of the universe only at late time. If the evolving trajectory of statefinder have a decelerating phase $(q>0)$ at early time, then we can understand why the expansion of the universe until $z \sim$ few remains unchanged in the scalar torsion models.

In this paper, we apply the statefinder diagnostics to the torsion cosmology. We find that there are some characteristics of statefinder parameters for the torsion cosmology that can be distinguished from the other cosmological models. The statefinder diagnostics show that the universe naturally has an accelerating expansion at low redshifts (late time) and a decelerating expansion at high redshifts (early time). Therefore, scalar torsion cosmology can avoid some of the problems which occur in other models. Especially, the effect of torsion can make the expansion rate oscillate when torsion parameter $a_{1}>0$ or $a_{1}<-1$. Whether the universe has properties which are easier to explain within the scalar torsion context is a remarkable possibility demanding further exploration. The oscillatory feature of Hubble parameter had earlier been reported for the braneworld cosmology [16] and the quasi-steady state cosmology [17]. We show that statefinder diagnostic has a direct bearing on the critical points of the dynamical system. One of the most interesting characteristic of the trajectories is that there are loop and curves with the shape of tadpole in the case of the torsion parameter $-\frac{1}{9} \leq a_{1}<0$. In this case, we fit the scalar torsion model to current type Ia 
supernova data and find it is consistent with the observations. Furthermore, we analyze preliminarily the relevance for realistic observation of the found statefinder parameters.

\section{The equations of motion}

PGT [18] has been regarded as an interesting alternative to general relativity because of its gauge structure and geometric properties. PGT based on a Riemann-Cartan geometry, allows for dynamic torsion in addition to curvature. The affine connection of the Riemann-Cartan geometry is

$$
\Gamma_{\mu \nu}{ }^{\kappa}=\bar{\Gamma}_{\mu \nu}{ }^{\kappa}+\frac{1}{2}\left(T_{\mu \nu}{ }^{\kappa}+T^{\kappa}{ }_{\mu \nu}+T^{\kappa}{ }_{\nu \mu}\right)
$$

where $\bar{\Gamma}_{\mu \nu}^{\kappa}$ is the Levi-Civita connection and $T_{\mu \nu}^{\kappa}$ is the torsion tensor. Meantime, the Ricci curvature and scalar curvature can be written as

$$
\begin{aligned}
R_{\mu \nu}= & \bar{R}_{\mu \nu}+\bar{\nabla}_{\nu} T_{\mu}+\frac{1}{2}\left(\bar{\nabla}_{\kappa}-T_{\kappa}\right)\left(T_{\nu \mu}{ }^{\kappa}+T_{\mu \nu}^{\kappa}+T_{\nu \mu}^{\kappa}\right) \\
& +\frac{1}{4}\left(T_{\kappa \sigma \mu} T^{\kappa \sigma}{ }_{\nu}+2 T_{\nu \kappa \sigma} T^{\sigma \kappa}{ }_{\mu}\right), \\
R= & \bar{R}+2 \bar{\nabla}_{\mu} T^{\mu}+\frac{1}{4}\left(T_{\mu \nu \kappa} T^{\mu \nu \kappa}+2 T_{\mu \nu \kappa} T^{\kappa \nu \mu}-4 T_{\mu} T^{\mu}\right),
\end{aligned}
$$

where $\bar{R}_{\mu \nu}$ and $\bar{R}$ are the Riemannian Ricci curvature and scalar curvature, respectively, and $\bar{\nabla}$ is the covariant derivative with the Levi-Civita connection (For a detailed discussion see Ref. [7].). Theoretical analysis of PGT led us to consider tendentiously dynamic "scalar mode". In this case, the restricted expression of the torsion can be written as [7]

$$
T_{\mu \nu \kappa}=\frac{2}{3} T_{[\mu} g_{\nu] \kappa}
$$

where the vector $T_{\mu} \equiv T_{\mu \nu}^{\nu}$ is the trace of the torsion. Then, the Ricci curvature and scalar curvature can be expressed as

$$
\begin{aligned}
R_{\mu \nu} & =\bar{R}_{\mu \nu}+\frac{1}{3}\left(2 \bar{\nabla}_{\nu} T_{\mu}+g_{\mu \nu} \bar{\nabla}_{k} T^{k}\right)+\frac{2}{9}\left(T_{\mu} T_{\nu}-g_{\mu \nu} T_{\kappa} T^{\kappa}\right) \\
R & =\bar{R}+2 \bar{\nabla}_{\mu} T^{\mu}-\frac{2}{3} T_{\mu} T^{\mu} .
\end{aligned}
$$

The gravitational Lagrangian density for the scalar mode is

$$
L=-\frac{a_{0}}{2} R+\frac{b}{24} R^{2}+\frac{a_{1}}{8}\left(T_{\nu \sigma \mu} T^{\nu \sigma \mu}+2 T_{\nu \sigma \mu} T^{\mu \sigma \nu}-4 T_{\mu} T^{\mu}\right),
$$

where $R \equiv R_{\mu \nu}^{\mu \nu}$ and $a_{1}$ is a torsion parameter. Consider that the parameter $b$ is associated with quadratic scalar curvature term $R^{2}$, so that $b$ should be positive [7]. Therefore, the field equations of the scalar mode are

$$
\begin{aligned}
& \bar{\nabla}_{\mu} R+\frac{2}{3}\left(R+\frac{6 \mu}{b}\right) T_{\mu}=0, \\
& a_{0}\left(\bar{R}_{\mu \nu}-\frac{1}{2} g_{\mu \nu} \bar{R}\right)=-\left(\mathcal{T}_{\mu \nu}+\widetilde{\mathcal{T}}_{\mu \nu}\right),
\end{aligned}
$$


where $\mathcal{T}_{\mu \nu}$ is the source energy-momentum tensor and $\widetilde{\mathcal{T}}_{\mu \nu}$ is the contribution of the scalar torsion mode to the effective total energy-momentum tensor:

$$
\begin{aligned}
\widetilde{\mathcal{T}}_{\mu \nu}= & -\frac{2 \mu}{3}\left(\bar{\nabla}_{(\mu} T_{\nu)}-g_{\mu \nu} \bar{\nabla}_{\kappa} T^{\kappa}\right)-\frac{\mu}{9}\left(2 T_{\mu} T_{\nu}+g_{\mu \nu} T_{\kappa} T^{\kappa}\right) \\
& -\frac{b}{6} R\left(R_{(\mu \nu)}-\frac{1}{4} g_{\mu \nu} R\right) .
\end{aligned}
$$

Since current observations favor a flat universe, we will work in the spatially flat Robertson-Walker metric $d s^{2}=-d t^{2}+a^{2}(t)\left[d r^{2}+r^{2}\left(d \theta^{2}+\sin ^{2} \theta d \phi^{2}\right)\right]$, where $a(t)$ is the scalar factor. This engenders the Riemannian Ricci curvature and scalar curvature:

$$
\begin{aligned}
& \bar{R}_{t}{ }^{t}=3 \frac{\ddot{a}}{a}=3\left(\dot{H}+H^{2}\right), \\
& \bar{R}_{r}{ }^{r}=\bar{R}_{\theta}{ }^{\theta}=\bar{R}_{\phi}{ }^{\phi}=\frac{\ddot{a}}{a}+2 \frac{\dot{a}^{2}}{a^{2}}=\dot{H}+3 H^{2}, \\
& \bar{R}=6\left(\frac{\ddot{a}}{a}+\frac{\dot{a}^{2}}{a^{2}}\right)=6\left(\dot{H}+2 H^{2}\right),
\end{aligned}
$$

where $a(t)$ is the scalar factor, and $H$ is the Hubble parameter. The torsion $T_{\mu}$ should also be only time dependent, therefore one can let $T_{t}(t) \equiv \Phi(t)(\Phi(t)$ is the torsion field) and the spatial parts vanish. The corresponding equations of motion in the matterdominated era are as follows

$$
\begin{aligned}
\dot{H} & =\frac{\mu}{6 a_{1}} R-\frac{\rho}{6 a_{1}}-2 H^{2}, \\
\dot{\Phi} & =-\frac{a_{0}}{2 a_{1}} R-\frac{\rho}{2 a_{1}}-3 H \Phi+\frac{1}{3} \Phi^{2}, \\
\dot{R} & =-\frac{2}{3}\left(R+\frac{6 \mu}{b}\right) \Phi,
\end{aligned}
$$

where $\mu=a_{1}-a_{0}$ and the energy density of matter component

$$
\rho=\frac{b}{18}\left(R+\frac{6 \mu}{b}\right)(3 H-\Phi)^{2}-\frac{b}{24} R^{2}-3 a_{1} H^{2} .
$$

One can scale the variables and the parameters as

$$
\begin{aligned}
& t \rightarrow l_{p}^{-2} H_{0}^{-1} t, H \rightarrow l_{p}^{2} H_{0} H, \Phi \rightarrow l_{p}^{2} H_{0} \Phi, R \rightarrow l_{p}^{4} H_{0}^{2} R, \\
& a_{0} \rightarrow l_{p}^{2} a_{0}, a_{1} \rightarrow l_{p}^{2} a_{1}, \mu \rightarrow l_{p}^{2} \mu, b \rightarrow l_{p}^{-2} H_{0}^{-2} b,
\end{aligned}
$$

where $H_{0}$ is the present value of Hubble parameter and $l_{p} \equiv \sqrt{8 \pi G}$ is the Planck length. Under the transform (2.18), Eqs. (2.14)-(2.16) remain unchanged. After transform, new variables $t, H, \Phi$ and $R$, and new parameters $a_{0}, a_{1}, \mu$ and $b$ are all dimensionless. Obviously, the Newtonian limit requires $a_{0}=-1$.

For the case of scalar torsion mode, the effective energy-momentum tensor can be represented as

$$
\begin{aligned}
& \widetilde{T}_{t}^{t}=-3 \mu H^{2}+\frac{b}{18}\left(R+\frac{6 \mu}{b}\right)(3 H-\Phi)^{2}-\frac{b}{24} R^{2}, \\
& \widetilde{T}_{r}{ }^{r}=\widetilde{T}_{\theta}^{\theta}=\widetilde{T}_{\phi}^{\phi}=\frac{1}{3}\left[\mu(R-\bar{R})-\widetilde{T}_{t}^{t}\right],
\end{aligned}
$$

and the off-diagonal terms vanish. The effective energy density

$$
\rho_{e f f}=\rho+\rho_{T} \equiv \rho+\widetilde{T}_{t t},
$$


which is deduced from general relativity. $p_{e f f}=p_{T} \equiv \tilde{T}_{r}^{r}$ is an effective pressure, and the effective equation of state is

$$
w_{e f f}=\frac{\widetilde{T}_{r}^{r}}{\rho+\widetilde{T}_{t t}}
$$

which is induced by the dynamic torsion.
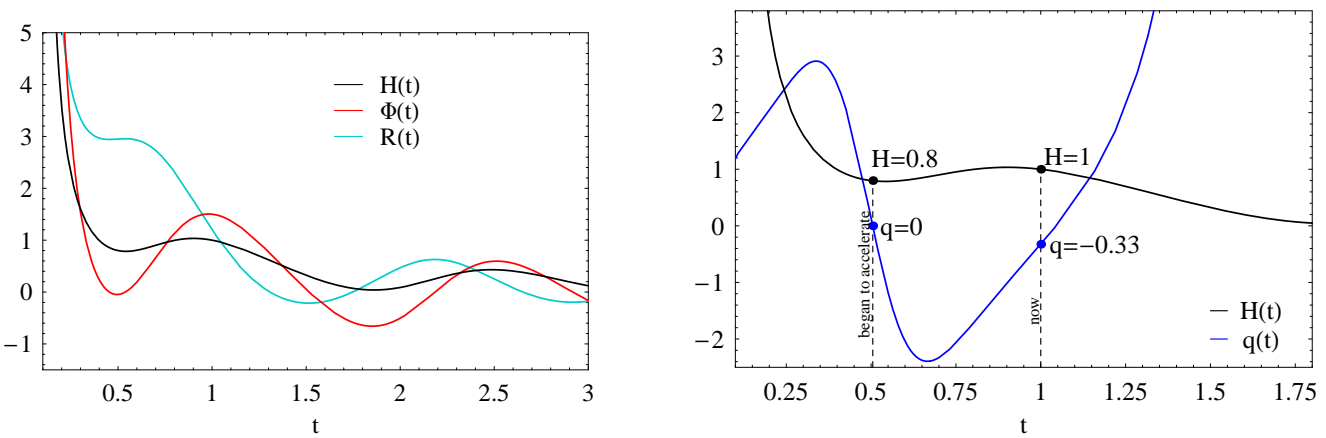

Figure 1. Evolution of the Hubble parameter $H$, the temporal component of the torsion $\Phi$, the affine scalar curvature $R$ and the deceleration parameter $q$ as functions of time. We have chosen the parameters $a_{1}=0.07, b=1.50$ and the initial values $H(1)=1, \Phi(1)=1.50, R(1)=1.20$.

In the case of $a_{1}>0$, Nester et al showed that the scalar mode can contribute an oscillating aspect to the expansion rate of the universe [7]. This oscillatory nature can be illustrated in Fig. 1 1 where we have chosen $a_{1}=0.07, b=1.50, H(1)=1$, $\Phi(1)=1.50, R(1)=1.20$ and set the current time $t_{0}=1$. According to scaling (2.18), the present value of the Hubble parameter is unity. Obviously, $H$ is damped-oscillating at late time and $q=-0.33$ is negative today, which means the expansion of the universe is currently accelerating. The value of $q$ turns from positive to negative when the time is around $t \approx 0.51$, which is the epoch the universe began to accelerate.

However, the above result is dependent on the choice of initial data and the values of the parameters. Then, the scalar torsion cosmology is unsuited to solving the finetuning problem in the case of $a_{1}>0$. In the following sections, we'll investigate the statefinder and give the dynamics analysis for all ranges of the parameter $a_{1}$.

\section{Statefinder diagnostic}

For the spatially flat $\Lambda$ CDM model the statefinder parameters correspond to a fixed point $\{1,0\}$ while $\{1,1\}$ for the standard cold dark matter model (SCDM) containing no radiation. Since the torsion cosmology have used the dynamic scalar torsion (a geometry quantity in the Riemann-Cartan spacetime), the torsion accelerating mechanism is bound to exhibit an essential distinction in contrast with various dark energy models. Therefore, its statefinder diagnostic is sure to reveal differential feature. Let us now study the torsion cosmological model in detail. Using Eqs. (2.14)-(2.17), we have the 
deceleration parameter

$$
q=\frac{1}{2}+\left[4(6 \mu+b R)(\Phi-3 H)^{2}-3 R(24 \mu+b R)\right]\left(432 a_{1} H^{2}\right)^{-1},
$$

and the statefinder parameters

$$
r=1+(6 \mu+b R)\left[b H\left(36 H^{2}-3 R+4 \Phi^{2}-24 H\right)-12 \Phi\right]\left(108 a_{1} b H^{3}\right)^{-1},
$$

and

$$
s=\frac{4(6 \mu+b R)\left[4 b H(3 H-\Phi)^{2}-3 b H R-12 \mu \Phi\right]}{3 b H\left[(6 \mu+b R)\left(36 H^{2}-24 H \Phi+4 \Phi^{2}-3 R\right)-54 \mu R\right]} .
$$

In the following we will discuss the statefinder for four differential ranges of the torsion parameter $a_{1}$, respectively. Firstly, we consider the time evolution of the statefinder pairs $\{r, s\}$ and $\{r, q\}$ in the case of $a_{1} \geq 0$. In Fig. 2, we plot evolution trajectories in the $q-r$ and $s-r$ planes, where we have chosen $a_{1}=1 / 2$ and $b=4$. We see easily that cosmic expansion alternates between deceleration and acceleration in the evolving trajectories of $q-r$ plane, and the amplitude becomes larger and larger as increase of time. The trajectories in the $s-r$ plane is quite complicated, so we mark its sequence by the ordinal number. Every odd number curve evolves from finite to infinite, but even number curve evolves from infinite to finite. These are quasi-periodic behaviors which corresponds to the numerical solution of Ref. [7]. Noticeably, the trajectories will never pass $\Lambda$ CDM point $\{1,0\}$.

Secondly, we discuss the time evolution of the statefinder pairs $\{r, s\}$ and $\{r, q\}$ for the case of $-\frac{1}{9} \leq a_{1}<0$. We plot evolving trajectories in Fig. 3, where we have chosen $a_{1}=-\frac{1}{10}$ and $b=3$. We see clearly that the cosmic acceleration is guaranteed by the dynamic scalar torsion in the evolving trajectories of $q-r$ plane, and the curves will converge into $\Lambda \mathrm{CDM}$ point. The evolving trajectories go through a climbing-up stage first, then get into a rolling-down stage in the $s-r$ plane. Lastly, trajectories tend to $\Lambda \mathrm{CDM}$ point $\{1,0\}$. Furthermore, the only one forms a loop that starts from $\{1,0\}$ then evolves back to $\{1,0\}$, and others show in the shape of tadpole.
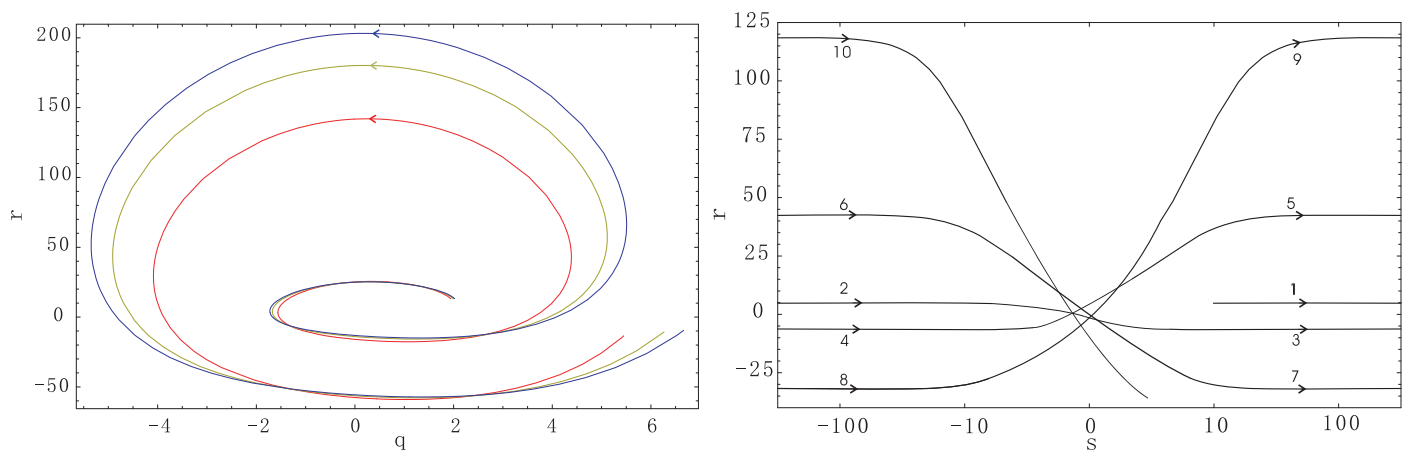

Figure 2. Evolving trajectories of the statefinder in the $q-r$ and $s-r$ planes for the case of torsion parameter $a_{1} \geq 0$, where we choose the parameters $a_{1}=\frac{1}{2}$ and $b=4$. The arrows show the direction of the time evolution. 

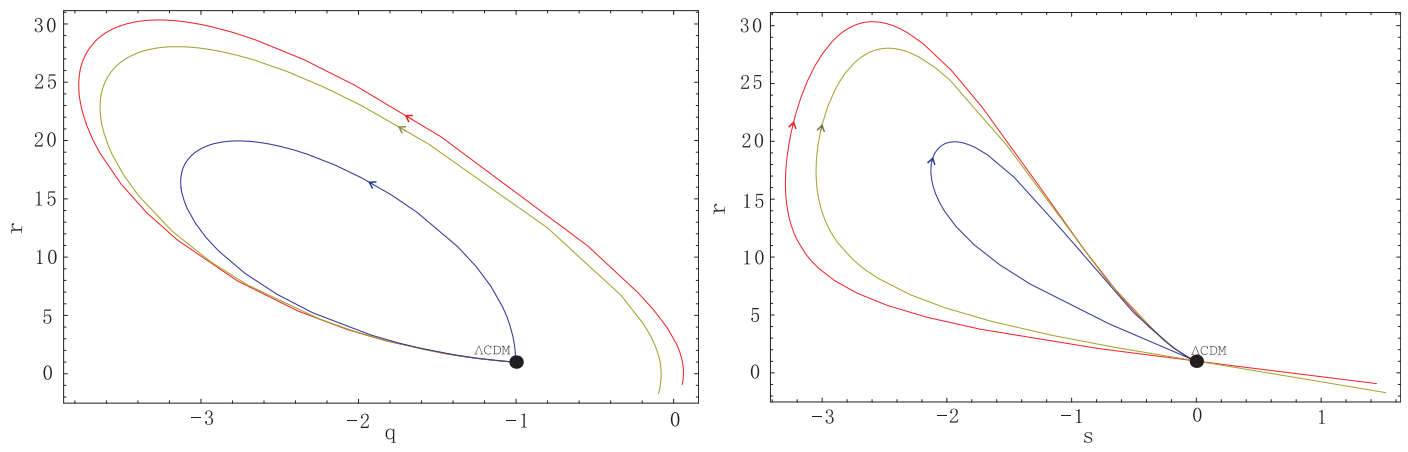

Figure 3. Evolving trajectories of the statefinder in the $q-r$ and $s-r$ planes for the case of torsion parameter $-\frac{1}{9} \leq a_{1}<0$, where we choose the parameters $a_{1}=-\frac{1}{10}$ and $b=3$. The arrows show the direction of the time evolution.
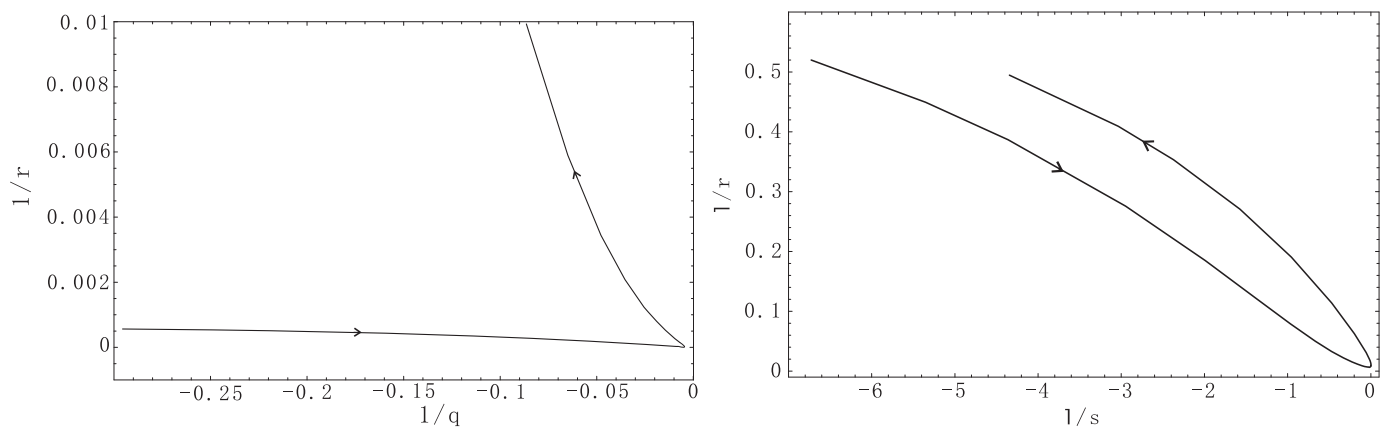

Figure 4. Evolving trajectories of the statefinder parameters in the $1 / q-1 / r$ and $1 / s-1 / r$ planes for the case of $-1 \leq a_{1}<-\frac{1}{9}$, where we choose $a_{1}=-\frac{1}{4}$ and $b=8$. The arrows show the direction of the time evolution.
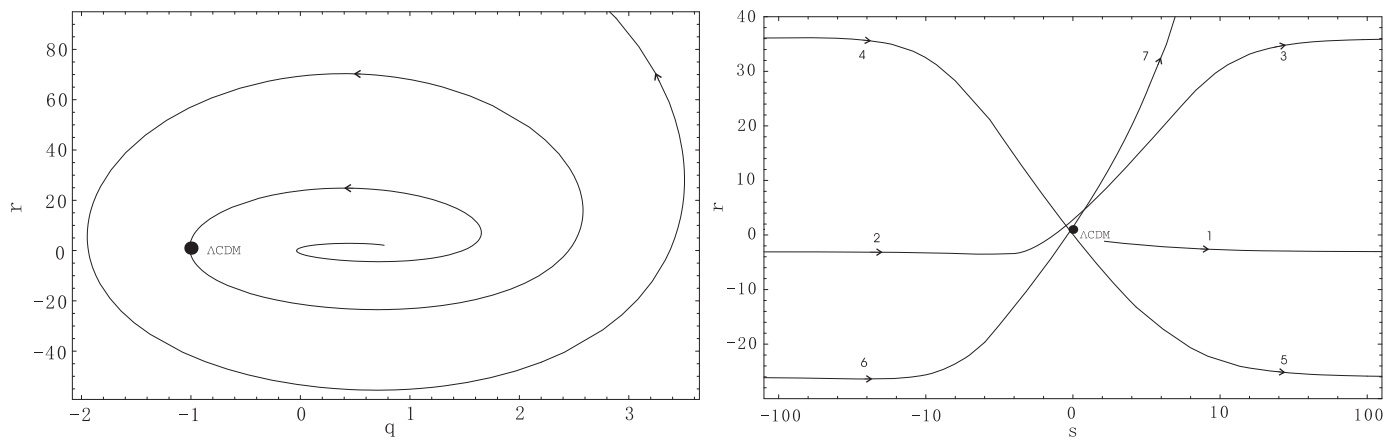

Figure 5. Evolving trajectories of the statefinder in the $q-r$ and $s-r$ planes for the case of torsion parameter $a_{1}<-1$, where we choose the parameters $a_{1}=-2$ and $b=4$. The arrows show the direction of the time evolution.

Thirdly, we discuss the time evolution of the trajectories for the case of $-1 \leq a_{1}<$ $-\frac{1}{9}$. We plot evolving trajectories in Fig. 目, where we have chosen $a_{1}=-\frac{1}{4}$ and $b=8$. Obviously, the cosmic acceleration can happen since deceleration parameter is negative. $|q|,|s|$ and $r$ become larger and larger first, then less and less as the cosmic time increase.

Finally, we consider the time evolution of the statefinder pairs $\{r, s\}$ and $\{r, q\}$ in the case of $a_{1}<-1$. In Fig. 5 , we plot evolving trajectories in the $q-r$ and $s-r$ planes, 
where we have chosen $a_{1}=-2$ and $b=4$. We find easily that the evolving trajectories analogous to the case of $a_{1} \geq 0$ except trajectories pass the $\Lambda \mathrm{CDM}$ point.

To sum up, it is very interesting to see that the scalar torsion naturally provide the accelerating force in the universe for any torsion parameter $a_{1}$. However, it is dependent on torsion parameters that there is a decelerating $(q>0)$ expansion before an accelerating $(q<0)$ expansion. The statefinder diagnostics show that the universe naturally has an accelerating expansion at low redshifts (late time) and a decelerating expansion at high redshifts (early time) for the cases of $-\frac{1}{9} \leq a_{1}$ and $a_{1}<-1$. Obviously, scalar torsion cosmology can avoid some of the problems which occur in other models. If we refuse the possibility of non-positivity of the kinetic energy, we will employ normal assumption, i. e., $a_{1}>0$. In this case, the effect of torsion can make the expansion rate oscillate. With suitable adjustments of the torsion parameters, it is possible to change the quasi-period of the expansion rate as well as its amplitudes. It is worth noting that the true values of the statefinder parameters of the universe should be determined in model-independent way. In principle, $r_{0}, s_{0}$ can be extracted from some future astronomical observations, especially the SNAP-type experiment.

Why there are new features for the statefinder diagnostic of torsion cosmology? Why the torsion parameter $a_{1}$ is divided into differential ranges by the statefinder diagnostic? Answer is very simple. In fact, the statefinder diagnostic has a direct bearing on the attractor of cosmological dynamics. Therefore, we will discuss the dynamic analysis in next section.

\section{Dynamics analysis}

Eqs. (2.14)-(2.16) is an autonomous system, so we can use the qualitative method of ordinary differential equations. Critical points are always exact constant solutions in the context of autonomous dynamical systems. These points are often the extreme points of the orbits and therefore describe the asymptotic behavior. If the solutions interpolate between critical points they can be divided into a heteroclinic orbit and a homoclinic orbit (a closed loop). The heteroclinic orbit connects two different critical points and homoclinic orbit is an orbit connecting a critical point to itself. In the dynamical analysis of cosmology, the heteroclinic orbit is more interesting [19]. If the numerical calculation is associated with the critical points, then we will find all kinds of heteroclinic orbits.

According to equations (2.14)-(2.16), we can obtain the critical points and study the stability of these points. Substituting linear perturbations $R=R_{c}+\delta R, \Phi=\Phi_{c}+\delta \Phi$ and $H=H_{c}+\delta H$ near the critical points into three independent equations, to the first orders in the perturbations, gives the evolution of the linear perturbations, from which we could yield three eigenvalues. Stability requires the real part of all eigenvalues to be negative. There are five critical points $\left(H_{c}, \Phi_{c}, R_{c}\right)$ of the system as follows

(i) $(0,0,0)$

(ii) $\left(\left(\frac{3\left(1+a_{1}\right)}{8}-A\right) \sqrt{B+C}, \frac{3}{2} \sqrt{B+C},-\frac{6\left(1+a_{1}\right)}{b}\right)$ 
Statefinder diagnostic in a torsion cosmology

$$
\begin{aligned}
& \text { (iii) }\left(-\left(\frac{3\left(1+a_{1}\right)}{8}-A\right) \sqrt{B+C},-\frac{3}{2} \sqrt{B+C},-\frac{6\left(1+a_{1}\right)}{b}\right) \\
& \text { (iv) }\left(\left(\frac{3\left(1+a_{1}\right)}{8}+A\right) \sqrt{B-C}, \frac{3}{2} \sqrt{B-C},-\frac{6\left(1+a_{1}\right)}{b}\right) \\
& \text { (v) }\left(-\left(\frac{3\left(1+a_{1}\right)}{8}+A\right) \sqrt{B-C},-\frac{3}{2} \sqrt{B-C},-\frac{6\left(1+a_{1}\right)}{b}\right)
\end{aligned}
$$

where $A=\frac{\sqrt{a_{1}^{2}\left(1+a_{1}\right)^{3}\left(1+9 a_{1}\right)}}{8 a_{1}\left(1+a_{1}\right)}, B=-\frac{\left(1+a_{1}\right)\left(5+9 a_{1}\right)}{a_{1} b}$ and $C=-\frac{3 \sqrt{a_{1}^{2}\left(1+a_{1}\right)^{3}\left(1+9 a_{1}\right)}}{a_{1}^{2} b}$. The corresponding eigenvalues of the critical points (i)-(v) are

$$
\begin{aligned}
& \text { (i) }\left(0,-\sqrt{-\frac{2\left(1+a_{1}\right)}{a_{1} b}}, \sqrt{-\frac{2\left(1+a_{1}\right)}{a_{1} b}}\right) \\
& \text { (ii) }\left(-\sqrt{B+C},-3\left(\frac{3\left(1+a_{1}\right)}{8}-A\right) \sqrt{B+C},-\left(\frac{1+9 a_{1}}{8}-3 A\right) \sqrt{B+C}\right) \\
& \text { (iii) }\left(\sqrt{B+C}, 3\left(\frac{3\left(1+a_{1}\right)}{8}-A\right) \sqrt{B+C},\left(\frac{1+9 a_{1}}{8}-3 A\right) \sqrt{B+C}\right) \\
& \text { (iv) }\left(-\sqrt{B-C},-3\left(\frac{3\left(1+a_{1}\right)}{8}+A\right) \sqrt{B-C},-\left(\frac{1+9 a_{1}}{8}+3 A\right) \sqrt{B-C}\right) \\
& \text { (v) }\left(\sqrt{B-C}, 3\left(\frac{3\left(1+a_{1}\right)}{8}+A\right) \sqrt{B-C},\left(\frac{1+9 a_{1}}{8}+3 A\right) \sqrt{B-C}\right)
\end{aligned}
$$

Using Eq. (4.1), we find that there is only a critical point $(0,0,0)$ in the case of $a_{1} \geq 0$. From Eq. (4.2), the corresponding eigenvalue is $\left(0,-\sqrt{\frac{2 \mu}{a_{1} b}} i, \sqrt{\frac{2 \mu}{a_{1} b}} i\right)$, so $(0,0,0)$ is an asymptotically stable focus. If we consider the linearized equations, then Eqs. (2.14)-(2.16) are reduced to

$$
\dot{H}=\frac{\mu}{6 a_{1}} R, \quad \dot{\Phi}=\frac{1}{2 a_{1}} R, \quad \dot{R}=-\frac{4 \mu}{b} \Phi .
$$

The linearized system (4.3) has an exact periodic solution

$$
\begin{aligned}
& H=-\alpha R_{0} \sin \omega t+\frac{\mu}{3} \Phi_{0} \cos \omega t+H_{0}-\frac{\mu}{3} \Phi_{0}, \\
& \Phi=-\beta^{-1} R_{0} \sin \omega t+\Phi_{0} \cos \omega t \\
& R=R_{0} \cos \omega t+\beta \Phi_{0} \sin \omega t
\end{aligned}
$$

where $\omega=-\sqrt{\frac{2 \mu}{\left|a_{1}\right| b}}, \alpha=\sqrt{\frac{b \mu}{72\left|a_{1}\right|}}, \beta=\sqrt{\frac{8 \mu\left|a_{1}\right|}{b}}$ and $H_{0}=H(0), \Phi_{0}=\Phi(0)$ and $R_{0}=R(0)$ are initial values. Obviously, $(H, 0,0)$ is a critical line of center for the linearized Eqs. (4.4). In other words, there are only exact periodic solutions for the linearized system, but there are quasi-periodic solutions near the focus for the coupled nonlinear equations. This property of quasi-periodic also appears in the statefinder diagnostic with the case of $a_{1} \geq 0$.

In the case of $-1 / 9 \leq a_{1}<0$, the critical point (ii) is a late time de Sitter attractor and (iii) is a negative attractor. The properties of the critical points are shown in table 1. The de Sitter attractor indicates that torsion cosmology is an elegant 
Table 1. The physical properties of critical points for $-\frac{1}{9} \leq a_{1}<0$

\begin{tabular}{llll}
\hline critical points & property & $w_{\text {eff }}$ & stability \\
\hline$($ i $)$ & saddle & $-\infty$ & unstable \\
$($ ii $)$ & positive attractor & -1 & stable \\
$($ iii $)$ & negative attractor & -1 & unstable \\
(iv) & saddle & -1 & unstable \\
(v) & saddle & -1 & unstable \\
\hline
\end{tabular}

Table 2. The physical properties of critical points for $a_{1}<-1$

\begin{tabular}{llll}
\hline critical points & property & $w_{\text {eff }}$ & stability \\
\hline$($ i $)$ & focus & $\pm \infty$ & stable \\
$($ ii $)$ & saddle & -1 & unstable \\
$($ iii $)$ & saddle & -1 & unstable \\
$($ iv $)$ & saddle & -1 & unstable \\
(v) & saddle & -1 & unstable \\
\hline
\end{tabular}

scheme and the scalar torsion mode is an interesting geometric quantity for physics. In the dynamical analysis of cosmology, the heteroclinic orbit is more interesting. Using numerical calculation, we plot the heteroclinic orbit connects the critical point case (iii) to case (ii) in Fig. 6. This heteroclinic orbit is just corresponding to the loop in Fig. 3. which is from $\Lambda \mathrm{CDM}$ point to $\Lambda \mathrm{CDM}$ point. Furthermore, the trajectories with the shape of tadpole correspond to saddles.

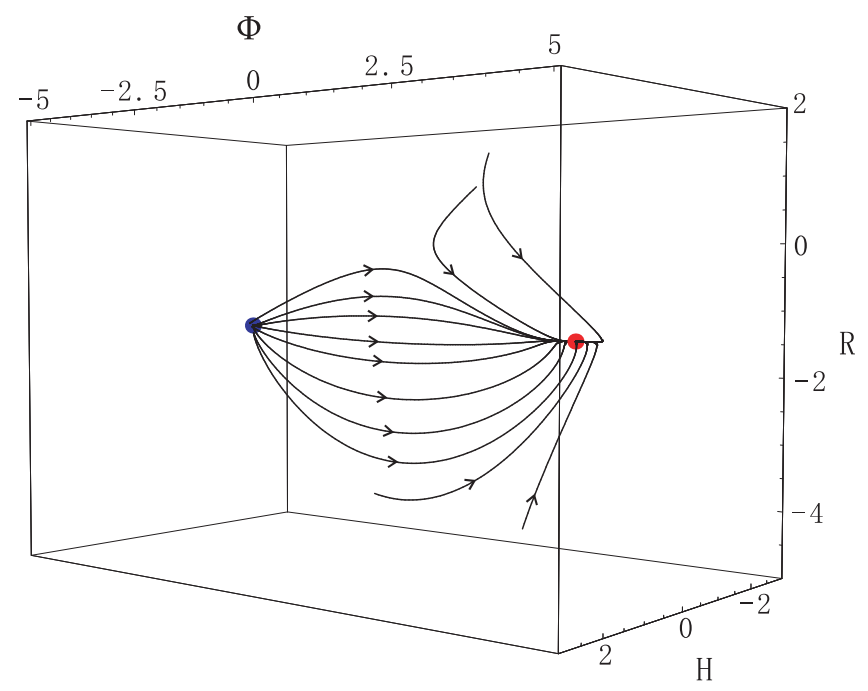

Figure 6. The phase diagrams of $(H, \Phi, R)$ with $-1 / 9 \leq a_{1}<0$. The heteroclinic orbit connects the critical points case (iii) to case (ii). We take $a_{1}=-1 / 10, b=4$. 


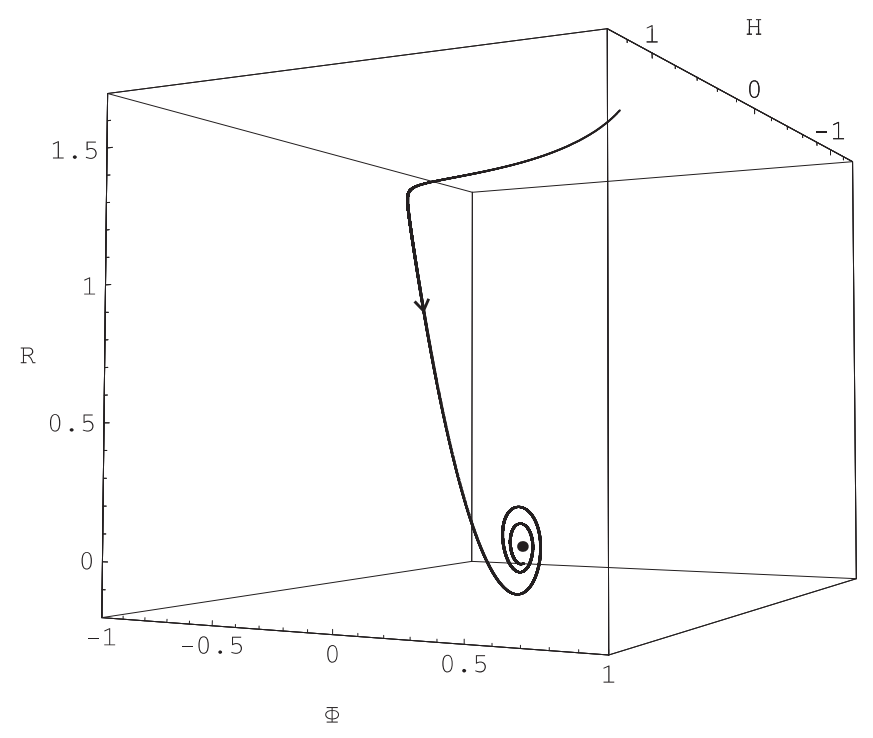

Figure 7. The phase diagrams of $(H, \Phi, R)$ with $a_{1}<-1$. We take $a_{1}=-2, b=4$ and the initial value $\left(5,1, \frac{29}{20}\right)$. $(0,0,0)$ is an asymptotically stable focus point.

In the case of $-1 \leq a_{1}<-\frac{1}{9}$, there is only an unstable saddle $(0,0,0)$ where the effective equation of state $w_{\text {eff }}$ tends to $-\infty$. Therefore, the trajectories in Fig. 团 show that $|q|,|s|$ and $r$ become larger and larger, then less and less as time increases.

In the case of $a_{1}<-1$, the properties of the critical points are shown in table 2 . The trajectories correspond to the stable focus (see Fig. 6) and unstable saddles with $w_{\text {eff }}=-1$. Therefore, the trajectories pass through the $\Lambda$ CDM point.

\section{Fit the torsion parameters to supernovae date}

In Ref. [7, the authors have compared the numerical values of the torsion model with the observational data, in which they fixed the initial values $H_{0}, \Phi_{0}$ and $R_{0}$, and torsion parameters $a_{1}$ and $b$. In this section, we fixed the initial value, then fit the torsion parameters to current type Ia supernovae data.

The scalar torsion cosmology predict a specific form of the Hubble parameter $H(z)$ as a function of redshifts $z$ in terms of two parameters $a_{1}$ and $b$ when we chose initial values. Using the relation between $d_{L}(z)$ and the comoving distance $r(z)$ (where $z$ is the redshift of light emission)

$$
d_{L}(z)=r(z)(1+z)
$$

and the light ray geodesic equation in a flat universe $c d t=a(z) d r(z)$ where $a(z)$ is the scale factor.

In general, the approach towards determining the expansion history $H(z)$ is to assume an arbitrary ansatz for $H(z)$ which is not necessarily physically motivated but is specially designed to give a good fit to the data for $d_{L}(z)$. Given a particular cosmological model for $H\left(z ; a_{1}, \ldots, a_{n}\right)$ where $a_{1}, \ldots, a_{n}$ are model parameters, the maximum likelihood technique can be used to determine the best fit values of parameters as well as the 
goodness of the fit of the model to the data. The technique can be summarized as follows: The observational data consist of $N$ apparent magnitudes $m_{i}\left(z_{i}\right)$ and redshifts $z_{i}$ with their corresponding errors $\sigma_{m_{i}}$ and $\sigma_{z_{i}}$. These errors are assumed to be gaussian and uncorrelated. Each apparent magnitude $m_{i}$ is related to the corresponding luminosity distance $d_{L}$ by

$$
m(z)=M+5 \log _{10}\left[\frac{d_{L}(z)}{\mathrm{Mpc}}\right]+25,
$$

where $M$ is the absolute magnitude. For the distant SNeIa, one can directly observe their apparent magnitude $m$ and redshift $z$, because the absolute magnitude $M$ of them is assumed to be constant, i.e., the supernovae are standard candles. Obviously, the luminosity distance $d_{L}(z)$ is the 'meeting point' between the observed apparent magnitude $m(z)$ and the theoretical prediction $H(z)$. Usually, one define distance modulus $\nu(z) \equiv m(z)-M$ and express it in terms of the dimensionless 'Hubble-constant free' luminosity distance $D_{L}$ defined by $D_{L}(z)=H_{0} d_{L}(z) / c$ as

$$
\nu(z)=5 \log _{10}\left(D_{L}(z)\right)+\nu_{0}
$$

where the zero offset $\nu_{0}$ depends on $H_{0}$ (or $h$ ) as

$$
\nu_{0}=5 \log _{10}\left(\frac{c H_{0}^{-1}}{\mathrm{Mpc}}\right)+25=-5 \log _{10} h+42.38 .
$$

The theoretically predicted value $D_{L}^{t h}(z)$ in the context of a given model $H\left(z ; a_{1}, \ldots, a_{n}\right)$ can be described by [20]

$$
D_{L}^{t h}(z)=(1+z) \int_{0}^{z} d z^{\prime} \frac{H_{0}}{H\left(z^{\prime} ; a_{1}, \ldots a_{n}\right)} .
$$

Therefore, the best fit values for the torsion parameters $\left(a_{1}, b\right)$ of the model are found by minimizing the quantity

$$
\chi^{2}\left(a_{1}, b\right)=\sum_{i=1}^{N} \frac{\left[\nu^{o b s}\left(z_{i}\right)-5 \log _{10} D_{L}^{t h}\left(z_{i} ; a_{1}, b\right)-\nu_{0}\right]^{2}}{\sigma_{i}^{2}} .
$$

Since the nuisance parameter $\nu_{0}$ is model-independent, its value from a specific good fit can be used as consistency test of the data [21] and one can choose a priori value of it (equivalently, the value of dimensionless Hubble parameter $h$ ) or marginalize over it thus obtaining

$$
\tilde{\chi}^{2}\left(a_{1}, b\right)=A\left(a_{1}, b\right)-\frac{B\left(a_{1}, b\right)^{2}}{C}+\ln \left(\frac{C}{2 \pi}\right),
$$

where

$$
\begin{aligned}
& A\left(a_{1}, b\right)=\sum_{i=1}^{N} \frac{\left[\nu^{o b s}\left(z_{i}\right)-5 \log _{10} D_{L}^{t h}\left(z_{i} ; a_{1}, b\right)\right]^{2}}{\sigma_{i}^{2}}, \\
& B\left(a_{1}, b\right)=\sum_{i=1}^{N} \frac{\left[\nu^{o b s}\left(z_{i}\right)-5 \log _{10} D_{L}^{t h}\left(z_{i} ; a_{1}, b\right)\right]}{\sigma_{i}^{2}}
\end{aligned}
$$


and

$$
C=\sum_{i=1}^{N} \frac{1}{\sigma_{i}^{2}}
$$

In the latter approach, instead of minimizing $\chi^{2}\left(a_{1}, b\right)$, one can minimize $\tilde{\chi}^{2}\left(a_{1}, b\right)$ which is independent of $\nu_{0}$.

The Eqs. 2.8 2.10) can be solved explicitly by a series in the form

$$
H\left(t, t_{0}\right)=H_{0}\left[1+\sum_{n=1}^{\infty} h_{n} u^{n}\right],
$$

where $u(t)=e^{\omega\left(t-t_{0}\right)}-1$ and

$$
\begin{aligned}
& h_{1}=-\frac{2 H_{0}}{\omega}-\frac{H_{0}}{2 \omega a_{1}}+\frac{R_{0}}{6 \omega H_{0}}+\frac{R_{0}}{6 \omega a_{1} H_{0}}-\frac{b H_{0} R_{0}}{12 \omega a_{1}}+\frac{b R_{0}^{2}}{144 \omega a_{1} H_{0}} \\
& +\frac{\Phi_{0}}{3 \omega}+\frac{\Phi_{0}}{3 \omega a_{1}}+\frac{b R_{0} \Phi_{0}}{18 \omega a_{1}}-\frac{\Phi_{0}^{2}}{18 \omega H_{0}}-\frac{\Phi_{0}^{2}}{18 \omega a_{1} H_{0}}-\frac{b R_{0} \Phi_{0}^{2}}{108 \omega a_{1} H_{0}}, \\
& h_{2}=\frac{H_{0}}{\omega}+\frac{H_{0}}{4 \omega a_{1}}-\frac{2 h_{1} H_{0}}{\omega}-\frac{h_{1} H_{0}}{2 \omega a_{1}}-\frac{b h_{1} R_{0} H_{0}}{12 \omega a_{1}}+\frac{b R_{0}^{2}}{288 \omega a_{1} H_{0}}+\frac{h_{1} \Phi_{0}}{6 \omega} \\
& +\frac{h_{1} \Phi_{0}}{6 \omega a_{1}}+\frac{b R_{0} \Phi_{0}}{36 \omega a_{1}}+\frac{b h_{1} R_{0} \Phi_{0}}{36 \omega a_{1}}-\frac{\Phi_{0}^{2}}{36 \omega H_{0}}-\frac{\Phi_{0}^{2}}{36 \omega a_{1} H_{0}}-\frac{b R_{0} \Phi_{0}^{2}}{108 \omega a_{1} H_{0}},
\end{aligned}
$$

Using the general relation between Hubble parameter $H(t)$ and the redshift $z, z$ can be written as a function of $t$

$$
z(t)=\exp \left[\int_{t}^{t_{0}} H(t) d t\right]-1
$$

However, the convergence radius of the series (5.11) is $\left|\frac{\ln 2}{\omega}\right|$, so we can use the expansion directly in the case of the redshift being $z<z\left(t_{0}+\frac{\ln 2}{\omega}\right)$. By the numerical calculation, we find that $t_{*} \sim t_{0}+\frac{\ln 2}{\omega}$ corresponds to $z_{*} \sim 0.45$ for the valuses of parameters $R_{0}$ and $\Phi_{0}$ in the Fig. 9. For the case of $z>z_{*}$, we should use a direct analytic continuation. Weierstrass [22] had built the whole theory of analytic functions from the concept of power series. Given a point $t_{1}=t_{*}+\alpha t_{*}(0<\alpha<1)$, the function $H(t)$ has a Taylor expansion

$$
H\left(t, t_{1}\right)=H\left(t_{1}, t_{0}\right)\left[1+\sum_{n=1}^{n} h_{n}\left(e^{\omega\left(t-t_{1}\right)}-1\right)^{n}\right] .
$$

where the coefficients $h_{n}$ is still expressed as Eq. (5.12) and $H\left(t_{1}, t_{0}\right)$ can be decided by Eq. (5.11). The new series defines an analytic function $H\left(t, t_{1}\right)$ which is said to be obtained from $H\left(t, t_{0}\right)$ by direct analytic continuation. This process can be repeated any number of times. In the general case we have to consider a succession of power series $H\left(t, t_{0}\right), H\left(t, t_{1}\right), \ldots, H\left(t, t_{m}\right)$, each of which is a direct analytic continuation of the preceding one. By using this method we have the evolution of Hubble parameter $H(t)$. Furthermore, we have the function $H(z)$ from Eq. (5.13). In fact, we need only to consider the case of $z<2$ for the ESSENCE supernovae data. 
We now apply the above described maximum likelihood method using the ESSENCE supernovae data which is one of the reliable published data set consisting of 192 SNeIa $(N=192)$. Beside the 162 data points given in table 9 of Ref. [23], which contains 60 ESSENCE SNeIa, 57 SNLS SNeIa and 45 nearby SNeIa, we add 30 SNeIa detected at $0.216<z<1.755$ by the Hubble Space Telescope [24] as in Ref. 25].

In table 3, we show the best fit of torsion parameters at different initial values of $R_{0}$ and $\Phi_{0}$. In Fig. 9, contours with $68.3 \%, 95.4 \%$ and $99.7 \%$ confidence level are plotted,

Table 3. The best fit of torsion parameters for different initial values of $R_{0}$ and $\Phi_{0}$

\begin{tabular}{llll}
\hline$R_{0}$ & $\Phi_{0}$ & $a_{1}$ & $b$ \\
\hline 0.25 & 0.35 & -0.10 & 1.44 \\
0.20 & 0.34 & -0.08 & 1.80 \\
0.15 & 0.34 & -0.06 & 2.40 \\
0.10 & 0.33 & -0.04 & 3.60 \\
\hline
\end{tabular}

in which we take a marginalization over the model-independent parameter $\nu_{0}$. The best fit as showed in the figure corresponds to $a_{1}=-0.06$ and $b=2.40$, and the minimum value of $\chi^{2}=355.68$. For $\Lambda C D M$, one can get $\chi^{2}(\Lambda C D M)=355.74$ and the best fit $\Omega_{m 0}=0.26$. Therefore, it's easy to know that $\Lambda C D M$ is consistent at the $1 \sigma$ level with the best fits of scalar torsion cosmology. In Fig. 8, we show a comparison of the ESSENCE supernovae data along with the theoretically predicted curves in the context of scalar torsion and $\Lambda C D M$. We can see that the scalar torsion $\operatorname{model}\left(R_{0}=0.15\right.$, $\left.\Phi_{0}=0.34, a_{1}=-0.06, b=2.4\right)$ gives a close curve behavior to the one from $\Lambda C D M$ $\left(\Omega_{m 0}=0.26, \Omega_{\Lambda}=0.74\right)$. Clearly, the allowed ranges of the parameters $a_{1}$ and $b$ favor the case of $-\frac{1}{9} \leq a_{1}<0$ if we chose $R_{0}=0.15$ and $\Phi_{0}=0.34$.
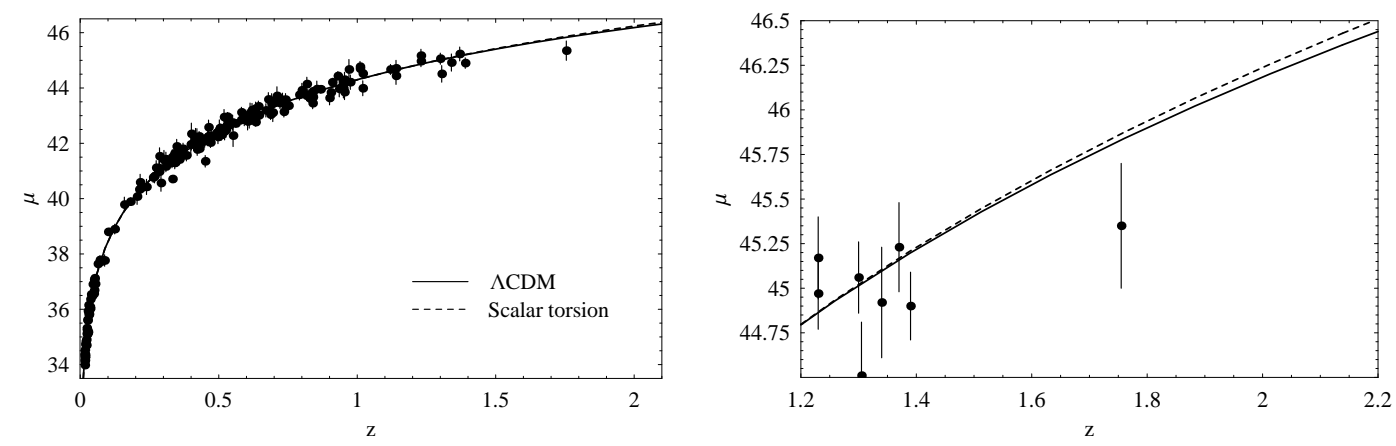

Figure 8. Comparison of scalar torsion model and $\Lambda C D M$ with the ESSENCE supernovae data via the relation between the redshift $z$ and the distance modulus $\mu$. The scalar torsion model $\left(R_{0}=0.15, \Phi_{0}=0.34, a_{1}=-0.06, b=2.4\right)$ gives a close curve behavior to the one from $\Lambda C D M\left(\Omega_{m 0}=0.26, \Omega_{\Lambda}=0.74\right)$. 


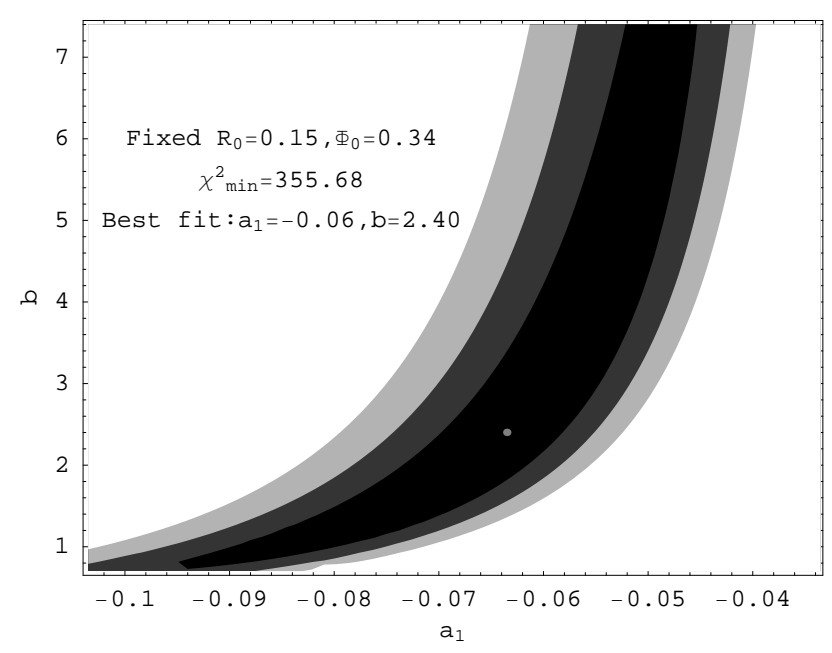

Figure 9. The $68.3 \%, 95.4 \%$ and $99.7 \%$ confidence contours of torsion parameters $a_{1}$ and $b$ using the ESSENCE SNeIa dataset. Here we have assumed $H_{0}=1, R_{0}=0.15$ and $\Phi_{0}=0.34$.

\section{Conclusion and discussion}

We have studied the statefinder diagnostic to the torsion cosmology, in which an accounting for the accelerated universe is considered in term of a Riemann-Cartan geometry: dynamic scalar torsion. We have shown that statefinder diagnostic has a direct bearing on the critical points. The statefinder diagnostic divides the torsion parameter $a_{1}$ into four ranges, which is in keeping with the requirement of dynamical analysis. Therefore, the statefinder diagnostic can be used to an exceedingly general category of models including several for which the notion of equation of state is not directly applicable. The statefinder diagnostic has the advantage over the dynamical analysis at the simplicity, but the latter can provide more information.

The most interesting characteristic of the trajectories is that there is a loop in the

case of $-\frac{1}{9} \leq a_{1}<0$. This behavior corresponds to the heteroclinic orbit connecting the negative attractor and de Sitter attractor. The trajectories with the shape of tadpole show that they pass through the $\Lambda \mathrm{CDM}$ fixed point along the time evolution, then the statefinder pairs are going along with a loop and they will pass through the $\Lambda \mathrm{CDM}$ fixed point again in the future. It is worth noting that there exists closed loop in the Ref. [14, but there is no closed loop which contains the $\Lambda \mathrm{CDM}$ fixed point. These behaviors indicate that torsion cosmology is an elegant scheme and the scalar torsion mode is an interesting geometric quantity for physics. Furthermore, the quasi-periodic feature of trajectories in the cases of $a_{1} \geq 0$ or $a_{1}<-1$ shows that the numerical solutions in Ref. [7] are not periodic, but are quasi-periodic near the focus for the coupled nonlinear equations.

We fixed only the initial values, then fitted the torsion parameters to current SNeIa dataset. We find that the scalar torsion naturally explain the accelerating expansion of the universe for any torsion parameter $a_{1}$. However, it is dependent $a_{1}$ and $b$ that 
there is a decelerating expansion before an accelerating expansion. The statefinder diagnostics show that the universe naturally have an accelerating expansion at late time and a decelerating expansion at early time for the case of $a_{1} \geq-\frac{1}{9}$ and $a_{1}<-1$. If we refuse the possibility of non-positivity of the kinetic energy, we have to employ normal assumption $\left(a_{1}>0\right)$. Under this assumption, the effect of torsion can make the expansion rate oscillate. Furthermore, with suitable adjustments of the torsion parameters and initial value, it is possible to change the quasi-period of expansion rate as well as its amplitudes. In order to have a quantitative understanding of the scalar torsion cosmology, the matter density $\rho$, the effective mass density $\rho_{\text {eff }}=\rho+\rho_{T}$, and the quantity $3 p_{T}+\rho_{\text {eff }}$ are important. This scenario bears a strong resemblance to the braneworld cosmology in a very different context by Sahni, Shtanov and Viznyuk [26]. The $\Omega_{m}$ parameters in the torsion cosmology and in the $\Lambda$ CDM cosmology can nevertheless be quite different. Therefore, at high redshift, the torsion cosmology asymptotically expands like a matter-dominated universe with the value of $\Omega_{m}$ inferred from the observations of the local matter density. At low redshift, the torsion model

behaves like $\Lambda$ CDM but with a renormalized value of $\Omega_{m}^{\Lambda C D M}$. The difference between $\Omega_{m}$ and $\Omega_{m}^{\Lambda C D M}$ is dependent on the present value of statefinder parameters $r_{0}, s_{0}$. A more detailed estimate, however, lies beyond the scope of the present paper, and we will study it in a future work. Finally, $r_{0}$ and $s_{0}$ should be extracted from some future astronomical observations in principle, especially the SNAP-type experiments.

\section{Acknowledgments}

This work is supported by National Science Foundation of China grant No. 10847153 and No. 10671128.

\section{References}

[1] Sahni V and Starobinsky A 2000 Int. J. Mod. Phys. D9 373 astro-ph/9904398

Peebles P J E and Ratra B 2003 Rev. Mod. Phys. 75559 astro-ph/0207347

Padmanabhan T 2003 Phys. Rept. 380235 hep-th/0212290

Copeland E J, Sami M and Tsujikawa S 2006 Int. J. Mod. Phys. D15 1753 hep-th/0603057

Sahni V and Starobinsky A 2006 Int. J. Mod. Phys. D15 2105 astro-ph/0610026

[2] Endo M and Fukui T 1977 Gen. Rel. Grav. 8833

Overduin J M and Cooperstock F I 1998 Phys. Rev. D58 043506 astro-ph/9805260

Hao J G and Li X Z 2005 Phys. Lett. B606 7 astro-ph/0404154

Liu D J and Li X Z 2005 Phys. Lett. B611 8 astro-ph/0501596

Beesham A 1993 Phys. Rev D48 3539

[3] Caldwell R R 2002 Phys. Lett. B545 23 astro-ph/9908168.

Sahni V and Shtanov Yu 2003 JCAP 11014 astro-ph/0202346

Hao J G and Li X Z 2003 Phys. Rev. D67 107303 gr-qc/0302100]

Liu D J and Li X Z 2003 Phys. Rev. D68 067301 hep-th/0307239

Li X Z and Hao J G 2004 Phys. Rev. D69 107303 hep-th/0303093

Hao J G and Li X Z 2003 Phys. Rev. D68 083514 hep-th/0306033

[4] Kerlick G D 1976 Ann. Phys. 99127 
[5] Goenner H and Müller-Hoissen F 1984 Class. Quant. Grav. 1651

[6] Capozziello S, Carloni S and Troisi A 2003 Recent Res. Dev. Astron. Astrophys. 1625 astro-ph/0303041

Boehmer C G and Burnett J 2008 Phys. Rev. D78 104001 [arXiv:0809.0469 [gr-qc]]

Boehmer C G 2005 Acta Phys. Polon. B36 2841 gr-qc/0506033

Mielke E W and Romero E S 2006 Phys. Rev. D73 043521

Minkevich A V, Garkin A S and Kudin V I 2007 Class. Quant. Grav. 245835 arXiv:0706.1157 [gr-qc]

[7] Shie K F, Nester J M and Yo H J 2008 Phys. Rev. D78 023522 arXiv:0805.3834 [gr-qc]]

Yo H J and Nester J M 2007 Mod. Phys. Lett. A22 2057 astro-ph/0612738

Li X Z, Sun C B and Xi P 2009 Phys. Rev. D79 027301 arXiv:0903.3088 [gr-qc]]

[8] Kopczyński W 1972 Phys. Lett. A39 219

[9] Nester J M and Isenberg J A 1977 Phys. Rev. D15 2078

[10] Hecht R D, Nester J M and Zhytnikov V V 1996 Phys. Lett. A222 37

Yo H J and Nester J M 1999 Int. J. Mod. Phys. D8 459 gr-qc/9902032

[11] Shapiro I L 2002 Phys. Rep. 357113 hep-th/0103093

[12] Hao J G and Li X Z 2004 Phys. Rev. D70 043529 [astro-ph/0309746.

[13] Sahni V, Saini T D, Starobinsky A A and Alam U 2003 JETP Lett. 77201 astro-ph/0201498

[14] Alam U, Sahni V and Saini T D and Starobinsky A A 2003 Mon. Not. Roy. Astron. Soc. 3441057 astro-ph/0303009

[15] Zhang X 2007 JCAP 0703007 gr-qc/0611084

Chang B, Liu H, Xu L, Zhang C and Ping Y 2007 JCAP 0701016 astro-ph/0612616

Liu D J and Liu W Z 2008 Phys. Rev. D77 027301 arXiv:0711.4854 [astro-ph]]

[16] Tretyakov P, Toporensky A, Shtanov Y and Sahni V 2006 Class. Quant. Grav. 233259 gr-qc/0510104

[17] Hoyel F, Burbidge G and Narlikar J V 1993 Astrophys. J. 410437 astro-ph/9412045

[18] Hehl F W, McCrea J D, Mielke E W and Neeman Y 1995 Phys. Rept. 2581 gr-qc/9402012

[19] Li X Z, Zhao Y B and Sun C B 2005 Class. Quant. Grav. 223759 astro-ph/0508019

[20] Starobinsky A A 1998 JETP Lett. 68757 astro-ph/9810431

Liu D J, Sun C B and Li X Z 2006 Phys. Lett. B634 442 astro-ph/0512355

[21] Choudury T R and Padmanabhan T 2005 Astron. Astrophys. 429807 [astro-ph/0311622]

[22] Ahlfors V 1979 Complex analysis (New York: McGraw Hill)

[23] Wood-Vasey W M et al. 2007 Astrophys. J 666694 astro-ph/0701041

[24] Riess A G et al. 2007 Astrophys. J 65998 astro-ph/0611572

[25] Davis T M et al. 2007 Astrophys. J 666716 [astro-ph/0701510

[26] Sahni V, Shtanov Y and Viznyuk A 2005 J. Cosmol. Astropart. Phys. 0512005 astro-ph/0505004 